\title{
EFEITOS DA POLÍTICA CAMBIAL SOBRE AS EXPORTAÇÕES DE CARNE BOVINA BRASILEIRA
}

\author{
THE EXPORTS OF BRAZILIAN BEEF AND THE EXCHANGE TAX
}

Isaac, F.I. ${ }^{1}$ e Souza, J.G. ${ }^{2}$

${ }^{1}$ Faculdade de Ciências Agrárias e Veterinárias. UNESP. Jaboticabal. SP. Brasil.

${ }^{2}$ Departamento de Economia Rural. Faculdade de Ciências Agrárias e Veterinárias. UNESP. Jaboticabal. São Paulo. Brasil. jgilbert@fcav.unesp.br

\section{PaLAVRAS ChaVE ADICIONAIS}

Carne bovina. Econometria. Economia. Exportação. Pecuária de corte.

\section{RESUMO}

O estudo teve como objetivo analisar a estrutura de mercado internacional de carne bovina e os efeitos da política cambial adotada pelo Brasil sobre as exportações e o preço da carne bovina brasileira, no período entre 1967 e 2003. Para se analisar o efeito da taxa de câmbio sobre os preços internacionais da carne bovina em moeda nacional, faz-se uso do método de análise conhecido por shift-share. Nessa análise, obtêmse os efeitos isolados das variações dos preços da carne bovina em dólares (efeito dólar) e da taxa de câmbio (efeito câmbio) sobre as variações dos preços em moeda nacional (efeito total). Verificase que a política cambial adotada pelo Brasil teve efeito positivo sobre as exportações de carne bovina no período entre 1967 e 2003. A taxa de câmbio real é claramente uma variável relevante para o desempenho do setor, corroborando resultados já obtidos por outros autores.

\section{SUMMARY}

This study aims at analyzing the structure of the international beef market and the effects of exchange rate policies employed by Brazil on the exports and the price of the Brazilian beef from 1967 through 2003. In order to analyze the effect of the exchange rate on international beef prices in Brazilian currency (real), the method known as shift-share is used. In that analysis, it is possible to obtain the isolated effect of the variations on beef prices in dollars (dollar effect) and on the

Recibido: 24-10-07. Aceptado: 14-5-08.

\section{AdDitional KeYWORDS}

Beef. Cattle. Econometrics. Economy. Export.

exchange rate (exchange rate effect) accross the variations of beef prices in national currency (total effect). The Brazilian exchange rate policy had a positive effect on the export of Brazilian beef from 1967 through 2003. Therefore, the real exchange rate plays a key role in the overall performance of the beef export sector, a result that has been obtained by other authors.

\section{INTRODUÇÃO}

Pode-se dizer que o Brasil tem aptidão para a produção de carne bovina; a extensão territorial e a localização geográfica favorecem o desenvolvimento de grande diversidade de gramíneas durante boa parte do ano, viabilizando economicamente a criação de gado para a produção de carne de qualidade, ou seja, o Brasil detém vantagens comparativas para a produção de carne bovina (ABIEC, 2005)

Fatores que alteram a quantidade exportada, como, por exemplo, renda per capita dos países compradores, preço da carne bovina, quantidade produzida pelo Brasil e seus concorrentes e a taxa de câmbio são variáveis que podem explicar o crescimento das exportações de carne do Brasil nos últimos anos (Ferreira, 2000; Miranda, 2001; Polaquini et al., 2006).

Arch. Zootec. 59 (225): 73-79. 2010. 
Este trabalho tem o propósito mensurar o efeito da taxa de câmbio sobre as exportações de carne bovina; e avaliar o efeito da taxa de câmbio real e dos preços internacionais em dólares sobre os preços da carne bovina em moeda nacional, no período entre 1967 e 2003.

\section{MATERIAL E MÉTODOS}

A taxa de câmbio real foi obtida usandose as taxas médias anuais do dólar comercial venda para o período em estudo, e foram corrigidas segundo o critério da paridade do poder de compra da moeda, que leva em conta a inflação doméstica e a inflação internacional, dada por:

$$
E=e^{*} / P
$$

onde:

$\mathrm{E}=$ taxa real de câmbio do Brasil (R\$/US\$);

$\mathrm{e}=$ taxa nominal de câmbio do Brasil (R\$/US\$), divulgada pelo IPEA;

$\mathrm{P}^{*}=$ índice de preços internacionais (WPI dos Estados Unidos);

$\mathrm{P}=$ índice de preços domésticos (WPI do Brasil).

A taxa de câmbio real (tabela I) permite medir o poder aquisitivo de uma economia e a competitividade da economia nacional em relação aos parceiros comerciais.

\section{MÉTOdo dE AVALIAÇÃO dO EFEITO DA TAXA DE CÂMBIO SOBRE OS PREÇOS DA CARNE BOVINA EM MOEDA NACIONAL}

O método de análise utilizado foi o shiftshare ou diferencial estrutural, muito usado em estudos para determinar taxas médias de variação e de crescimento na agricultura. Este método foi utilizado por Curtis (1972), Camargo (1983), Mendonça de Barros et al. (1983), Yokoyama et al. (1989), Campos (1991), Silva e Carvalho (1995) e Reis (1997).

Para Silva e Carvalho (1995), essa metodologia permite dimensionar a importância relativa da variação cambial sobre os preços entre dois momentos no tempo.
Tabela I. Decomposição da taxa anual de crescimento do preço da carne bovina em moeda nacional, 1967 a 2003. (Analysis of the annual rate of growth in beef prices in national currency, from 1967 to 2003).

\begin{tabular}{|c|c|c|c|}
\hline Ano & Total & $\begin{array}{l}\text { Efeito } \\
\text { dólar }\end{array}$ & Câmbio \\
\hline 1967 & - & - & - \\
\hline 1968 & $-1,39$ & $-6,94$ & 5,56 \\
\hline 1969 & $-4,10$ & $-7,07$ & 2,97 \\
\hline 1970 & 14,75 & 19,68 & $-4,93$ \\
\hline 1971 & 60,67 & 62,83 & $-2,16$ \\
\hline 1972 & $-4,85$ & $-2,77$ & $-2,08$ \\
\hline 1973 & 32,34 & 37,36 & $-5,01$ \\
\hline 1974 & 24,70 & 25,89 & $-1,19$ \\
\hline 1975 & $-13,40$ & $-16,85$ & 3,46 \\
\hline 1976 & $-3,25$ & 1,32 & $-4,57$ \\
\hline 1977 & $-6,41$ & $-5,65$ & $-0,76$ \\
\hline 1978 & 12,08 & 11,72 & 0,36 \\
\hline 1979 & 62,12 & 53,64 & 8,48 \\
\hline 1980 & 25,03 & 15,63 & 9,40 \\
\hline 1981 & $-16,80$ & $-10,30$ & $-6,50$ \\
\hline 1982 & $-19,74$ & $-22,72$ & 2,98 \\
\hline 1983 & 13,86 & $-6,62$ & 20,47 \\
\hline 1984 & $-5,70$ & $-2,12$ & $-3,58$ \\
\hline 1985 & $-0,97$ & $-4,25$ & 3,28 \\
\hline 1986 & $-4,47$ & 5,84 & $-10,31$ \\
\hline 1987 & 30,32 & 35,75 & $-5,43$ \\
\hline 1988 & $-34,20$ & $-23,85$ & $-10,36$ \\
\hline 1989 & $-23,10$ & $-5,94$ & $-17,16$ \\
\hline 1990 & $-11,26$ & $-1,49$ & $-9,77$ \\
\hline 1991 & 12,19 & 36,32 & $-24,12$ \\
\hline 1992 & 49,90 & $-5,55$ & 55,45 \\
\hline 1993 & $-10,14$ & $-1,14$ & $-9,00$ \\
\hline 1994 & $-3,21$ & 18,18 & $-21,40$ \\
\hline 1995 & 10,97 & 21,03 & $-10,06$ \\
\hline 1996 & $-5,06$ & $-10,45$ & 5,39 \\
\hline 1997 & $-4,99$ & $-4,88$ & $-0,11$ \\
\hline 1998 & 3,36 & 0,38 & 2,98 \\
\hline 1999 & 17,54 & $-14,14$ & 31,68 \\
\hline 2000 & $-18,83$ & $-8,13$ & $-10,70$ \\
\hline 2001 & $-3,16$ & $-16,85$ & 13,69 \\
\hline 2002 & $-2,99$ & $-7,61$ & 4,62 \\
\hline 2003 & $-12,40$ & 2,90 & $-15,30$ \\
\hline Média & 4,43 & 4,53 & $-0,10$ \\
\hline
\end{tabular}

Archivos de zootecnia vol. 59, núm. 225, p. 74. 


\section{Metodologia PARA deCOMPOSIÇÃo do} EFEITO PREÇO INTERNACIONAL EM EFEITO DÓLAR E EFEITO CÂMBIO

Neste estudo, foi utilizada a adaptação do modelo proposto por Reis (1997), em que se decompõem as variações no preço internacional da carne bovina em moeda nacional nos efeitos preço internacional em dólares (efeito dólar) e o efeito da taxa de câmbio (efeito câmbio), para o período compreendido entre 1967 e 2003.

Foi obtida a taxa de crescimento anual dos preços internacionais da carne bovina em moeda nacional. Essa taxa será a variação ocorrida entre o ano analisado em relação ao ano anterior.

A expressão [2] representa a conversão do preço da carne bovina em reais, em um dado período "t";

$$
P R_{t}=P D_{t} * E_{t}
$$

onde:

$\mathrm{PR}_{\mathrm{t}}=$ preço da carne bovina em reais:

$\mathrm{PD}_{\mathrm{t}}=$ preço da carne bovina em dólares;

$E_{t}=$ taxa de câmbio real do Brasil (R\$/US\$).

Os períodos inicial e final são indicados pelos índices "0" e "t", respectivamente.

De forma idêntica à expressão [2], a expressão [3] mostra o preço em reais, no período inicial "0";

$$
P R_{0}=P D_{0}{ }^{*} E_{0}
$$

A expressão [4] mostra o preço em reais quando ocorre somente alteração no preço em dólares;

$$
P R_{t}^{P D}=P D_{t}^{*} E_{0}
$$

A expressão [5] mostra o preço em reais quando somente a taxa de câmbio varia;

$$
P R_{t}^{E}=P D_{0} * E_{t}
$$

A mudança no preço em reais entre o período "0" e o período "t" é expressa por:

$$
P R_{t}-P R_{0}=\left(P R_{t}^{P D}-P R_{0}\right)+\left(P R_{t}-P R_{t}^{P D}\right)
$$

onde:

$P R_{t}-P R_{0}=$ variação total no preço em reais; $P R_{t}^{P D}-P R_{0}=$ efeito preço internacional em dólares; $P R_{t}-P R_{t}^{P D}=$ efeito câmbio.

Segundo Igreja, citado por Campos (1991), os efeitos explicativos podem ser apresentados individualmente na forma de taxas de crescimento que, somadas, resultarão na taxa média anual de crescimento.

Então, usando-se a expressão [7] e multiplicando-se ambos os lados dessa expressão por:

$$
1 /\left(P R_{t}-P R_{0}\right)
$$

tem-se:

$1=\left(\mathrm{PR}_{\mathrm{t}}^{\mathrm{PD}}-\mathrm{PR}_{0}\right) /\left(\mathrm{PR}_{\mathrm{t}}-\mathrm{PR}_{0}\right)+\left(\mathrm{PR}_{\mathrm{t}}-\mathrm{PR}_{\mathrm{t}}^{\mathrm{PD}}\right) /\left(\mathrm{PR}_{\mathrm{t}}-\mathrm{PR}_{0}\right)$

A seguir, multiplicando-se ambos os lados da identidade [8] por

$$
\begin{aligned}
& r=\left(\sqrt[1]{P R_{\mathrm{t}} / P R_{0}}-1\right) * 100 \\
& \text { se } t=1, \text { tem-se } \\
& r=\left(P R_{t} / P R_{0}-1\right) * 100
\end{aligned}
$$

onde:

$r=$ taxa média anual de variação do preço em reais, em porcentagem ano (efeito total).

Então, obtém-se:

$r=\left(P R_{t}^{P D}-P R_{0}\right)^{*} r /\left(P R_{t}-P R_{0}\right)+\left(P R_{t}-P R_{t}^{P D}\right) * r /\left(P R_{t}^{-}\right.$ $P R_{0}$ )

onde:

$\left(P R_{t}^{P D}-P R_{0}\right)^{*} /\left(P R_{t}-P R_{0}\right)=$ efeito preço internacional em dólares, expresso em porcentagem ano (efeito dólar);

$\left(P R_{t}-P R_{t}{ }^{P D}\right){ }^{*} /\left(P R_{t}-P R_{0}\right)=$ efeito câmbio, expresso em porcentagem ano (efeito câmbio).

Archivos de zootecnia vol. 59, núm. 225, p. 75. 


\section{RESULTADOSEDISCUSSÃO}

\section{EFEITO DA TAXA DE CÂMBIO SOBRE AS EXPORTAÇÕESDECARNEBOVINA}

Para essa análise, fez-se uso da equação de oferta de exportação, em que se observa ter sido de 2,002 o valor do coeficiente estimado para a variável taxa de câmbio real do Brasil, sendo considerado como elasticidade-câmbio (Isaac, 2006). Assim, podese afirmar que, para uma variação de $10 \%$ na taxa de câmbio real, ceteris paribus, haverá uma variação de aproximadamente $20 \%$ nas quantidades exportadas de carne bovina do Brasil, no mesmo sentido.

Diante disso, observa-se o efeito positivo da taxa de câmbio sobre as exportações de carne bovina do Brasil. Isso significa que, quando a moeda nacional está desvalorizada em relação ao dólar (taxa de câmbio alta), maior é o incentivo às exportações, ocorrendo o inverso quando a moeda nacional está valorizada (taxa de câmbio baixa).

A partir da segunda metade da década de 1990, com a implantação do Plano Real, observa-se queda nas exportações de carne bovina e aumento do consumo interno, tendo sido estes efeitos causados pelo aumento na renda do consumidor.

A queda nas exportações a partir de 1994 também se deve pelo encarecimento da carne bovina do Brasil, em dólares, causado pela valorização da moeda nacional em relação ao dólar.

\section{EFEITODA TAXA DE CÂMBIO REAL DO BRASIL} E DO PREÇO DE EXPORTAÇÃO DA CARNE BOVINA SOBRE OS PREÇOS EM MOEDA NACIONAL

A valorização ou desvalorização de uma moeda pode influenciar positiva ou negativamente os preços domésticos de um produto. Para verificar essa influência, buscou-se analisar os efeitos da taxa de câmbio sobre os preços de exportação da carne bovina em moeda nacional entre 1967 e 2003.

Considerando-se que o preço de exportação da carne bovina em moeda nacional pode ser obtido pela multiplicação do preço de exportação em dólares pela taxa de câmbio real adotada pelo Brasil (R\$/US\$), qualquer

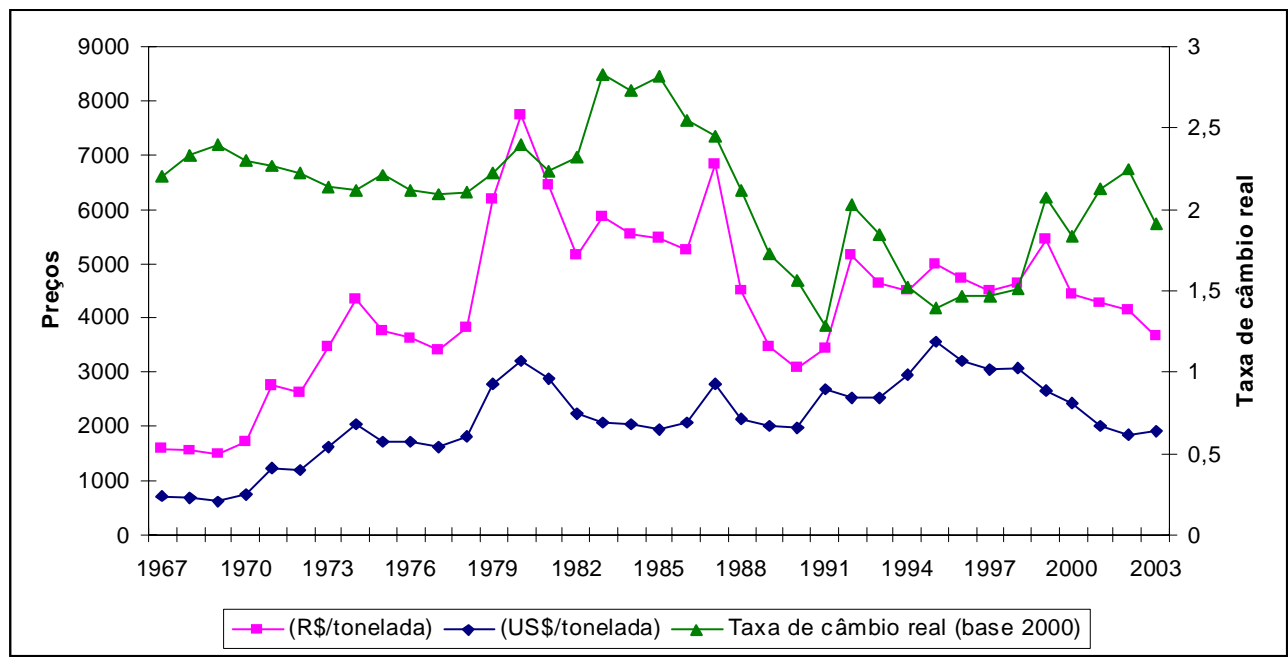

Figura 1. Comportamento dos preços médios anuais da carne bovina em reais e em dólares e da taxa média anual de câmbio real do Brasil, 1967 a 2003. (Evolution of average annual prices of beef in reais and in dollars and the average annual real exchange rate in Brazil, from 1967 to 2003).

Archivos de zootecnia vol. 59, núm. 225, p. 76. 


\section{APOLÍTICA CAMBIAL E AS EXPORTAÇÕES DE CARNE BOVINA BRASILEIRA}

variação isolada ou em conjunto nesses fatores pode afetar os preços de exportação.

A figura 1 mostra que, durante o período em estudo, tanto os preços de exportação como a taxa de câmbio apresentaram-se com oscilações. O mesmo acontece com os preços em moeda nacional (em reais), que sofreram grande influência dessas variações.

Observa-se também que o preço da carne bovina em moeda nacional vem caindo desde 1999. O comportamento dos preços médios anuais, em moeda nacional, deve-se ao efeito conjunto das variações ocorridas nos preços médios anuais em dólares e na taxa média de câmbio real.

Geralmente, nos anos em que o preço da carne bovina em moeda nacional apresentouse maior que o preço médio do período analisado, o preço em dólares e a taxa de câmbio também se apresentaram acima da média do período analisado. O mesmo podese dizer quando os preços encontraram-se abaixo da média do período analisado.

De acordo com Silva e Carvalho (1995), a variação real do câmbio tanto pode elevar como reduzir a variabilidade dos preços em moeda nacional. Aumentará a variabilidade se, predominantemente, nos períodos em que a moeda estiver valorizada, os preços em dólares estiverem em queda e vice-versa. Se acontecer o inverso, a instabilidade será menor quando calculada sobre o preço em moeda nacional.

Para verificar a variabilidade dos preços em moeda nacional, foi incluído nesta análise o valor do coeficiente de variação dos preços.

Analisando-se o coeficiente de variação (C.V.), pode-se observar que os preços em moeda nacional tiveram pouca instabilidade (C.V.= 34,15\%). Essa variação decorreu em maior proporção em razão do efeito dólar (C.V.= 35,67\%) e em menor proporção ao efeito câmbio (C.V.=18,66\%), pois o preço em dólar apresentou maior coeficiente de variação que a taxa de câmbio real (tabela I).

Na tabela I, mostram-se os resultados obtidos na decomposição dos preços internacionais da carne bovina em moeda nacional. Observa-se que ocorreram grandes variações dos preços internacionais da carne bovina em moeda nacional quando se calculou as taxas anuais de crescimento (efeito total).

A maior variação ocorreu em 1979, quando a taxa anual de crescimento chegou a 62,12\% em relação a 1978. Esse aumento no preço em moeda nacional teve como principal contribuição o aumento do preço em dólar (efeito dólar), que variou cerca de $53,6 \%$, acompanhado de uma variação de aproximadamente $8,5 \%$ na taxa de câmbio (efeito câmbio).

A maior queda foi registrada em 1988 , quando o efeito total chegou a -4,2\%. Essa taxa negativa foi influenciada principalmente pelo efeito dólar, que foi de -23,85 e também pelo efeito câmbio, que foi de-10,36.

Desde o final da década de 1960 até o início da década de 1980, o efeito dólar foi o fator que mais influenciou os preços da carne bovina em moeda nacional. A partir de então, o efeito câmbio passou a influenciar um pouco mais os preços da carne bovina em moeda nacional.

O efeito câmbio teve grande relevância no início da década de 1990. Em 1994, o efeito câmbio negativo fez os preços em moeda nacional apresentarem variações negativas, contrapondo-se ao efeito dólar positivo. Em 1992 e 1999, ocorreu o inverso, o efeito câmbio positivo levou a variações positivas no preço em moeda nacional quando o efeito dólar foi negativo.

As variações reais na taxa de câmbio têm impacto expressivo sobre a competitividade externa do produto exportado. No caso da carne bovina, que é um produto agrícola, variações reais no câmbio somam-se aos efeitos dos subsídios de países desenvolvidos, que distorcem os preços internacionais. A competitividade não depende somente dos preços, mas este é um dos mais importantes componentes.

Os efeitos de câmbio negativo mais significantes mostraram que, mesmo estan-

Archivos de zootecnia vol. 59, núm. 225, p. 77. 
do alto o preço da carne bovina em dólares em determinados anos, os exportadores não usufruíram desse aumento, pois a moeda nacional encontrava-se valorizada, resultando em preços mais baixos no mercado interno.

A figura 2 ilustra como a taxa de câmbio real e o volume de carne bovina exportado pelo Brasil variaram entre 1967 e 2003.

Nota-se que quando há desvalorização da moeda nacional, ocorre aumento no volume exportado, é o que ocorre mais claramente durante a década de 1980, início da década de 1990 e a partir de 1998.

\section{CONCLUSÕES}

Considerando-se que o topo do ranking dos países exportadores de carne bovina foi alcançado pelo Brasil com uma parcela pequena de sua produção e com índices zootécnicos considerados baixos, pode-se inferir que muito ainda deve ser feito para, definitivamente, consolidar o Brasil como o maior exportador de carne bovina do mun- do. Os dados apresentados neste trabalho evidenciam o potencial do país para comercialização de carne bovina.

Quando se leva em consideração a política cambial adotada pelo Brasil, verifica-se que ela teve efeito positivo sobre as exportações de carne bovina no período entre 1967 e 2003. A taxa de câmbio real é claramente uma variável relevante para o desempenho do setor, corroborando resultados já obtidos por outros autores.

Atente-se que as desvalorizações cambiais, além de beneficiarem os exportadores de carne bovina, gerando maior possibilidade de receita com as vendas externas, são também um instrumento para melhorar sua competitividade no mercado internacional.

Esta afirmação decorre do fato de que o fator preço é um instrumento usado entre os concorrentes nesse mercado para conquistar maior participação. A possibilidade de reduzir preços de exportação em dólar, decorrente da desvalorização cambial ou da redução dos preços do boi gordo (matéria-

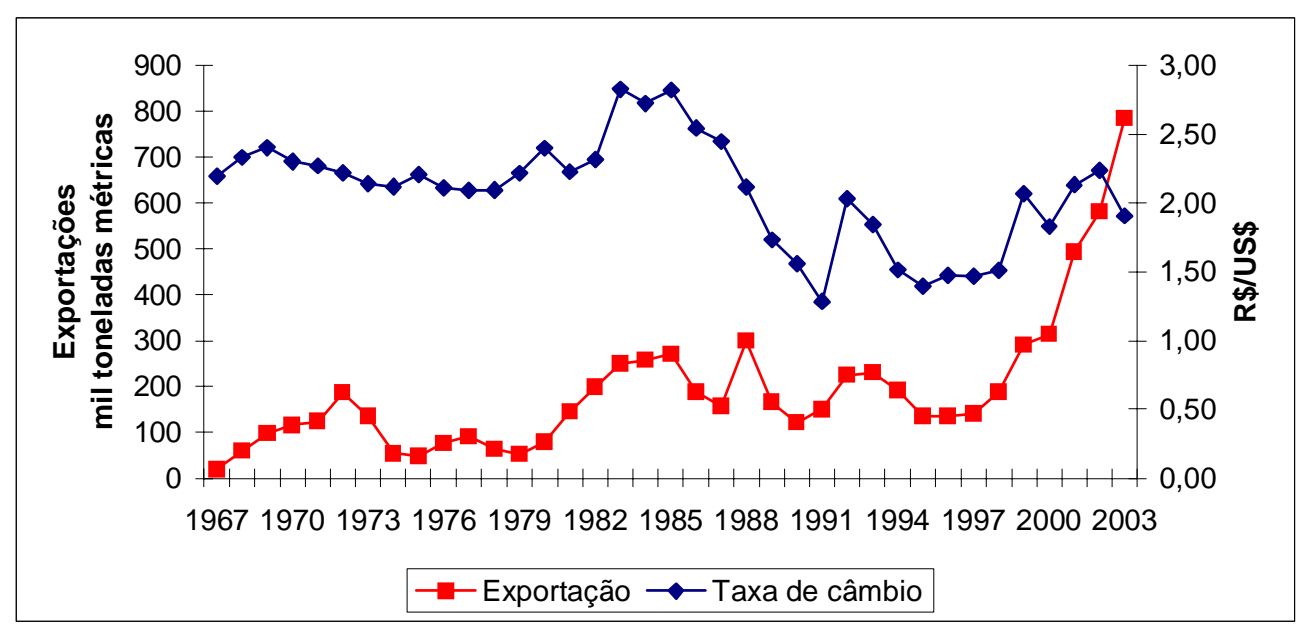

Fonte:FAO/ONU, IPEA E FMI. Organizado pelos autores.

Figura 2. Variação da taxa de câmbio real e volume de carne bovina exportada pelo Brasil, entre 1967 e 2003. (Change in real exchange rate and volume of beef exported by Brazil, between 1967 and 2003).

Archivos de zootecnia vol. 59, núm. 225, p. 78. 


\section{A POLÍTICA CAMBIAL EAS EXPORTAÇÕES DE CARNE BOVINA BRASILEIRA}

prima básica) é, um fator de competitividade nesse mercado.

Os preços da carne bovina em moeda nacional apresentaram tendência de queda a partir de meados da década de 1990 e os preços em dólar mostraram comportamento semelhante. Tal fato revela que os preços em dólares têm maior influência sobre os preços em reais que a taxa de câmbio do período. O efeito dólar é mais significativo que o efeito câmbio sobre os preços em moeda nacional.

Embora o valor da moeda nacional em relação ao dólar seja um fator importante para a competitividade brasileira no mercado internacional, as políticas setoriais que

\section{BIBLIOGRAFIA}

ABIEC. 2005. Associação Brasileira das Indústrias Exportadoras de Carnes. Balanço da pecuária bovídea de corte. Disponível em: <http:// www.abiec.com.br>. Acesso em (20 jun 2005).

Camargo, A.M.M.P. 1983. Substituição regional entre as principais atividades agrícolas no Estado de São Paulo. Piracicaba, 1983. Dissertação (Mestrado). Escola Superior de Agricultura Luiz de Queiroz. Universidade de São Paulo. Piracicaba. 263 pp.

Campos, R.T. 1991. Efeitos do ataque do bicudo na cotonicultura do semi-árido cearense. Tese (Doutorado). Universidade Federal de Pernambuco. Recife. 160 pp.

Curtis, W.C. 1972. Shift-share analysis as a technique in rural development research. Am. J. Agr. Econ., 54: 267-70.

Ferreira, R.C. 2000. Entraves comerciais às exportações de carne bovina. Informe Agropecuário, 21, nº 205. jul./ago. 2000.

Isaac, F.I. 2006. Política cambial e exportação de carne no Brasil. Dissertação (Mestrado em Zootecnia). Universidade Estadual Paulista Júlio de Mesquita Filho. Jaboticabal-SP. Brasil.

Mendonça de Barros, J.R de, Pastore, A.F. e Rizzieri, J.A.B. 1983. A evolução recente da agricultura brasileira até 1970. Em: Araújo, P.F.C. propiciam aumento da produtividade e queda no preço se constituem no melhor instrumento para se obter excedentes exportáveis, isto, por terem caráter mais independente do que as políticas macroeconômicas, que afetam muitos segmentos da economia. (Polaquini et al., 2006).

A adoção de medidas como incentivo ao uso de tecnologia e aumento de produtividade nas fazendas, políticas de melhoria da qualidade do produto ofertado e uma ação de marketing eficiente que divulgue a qualidade da carne bovina do Brasil no exterior são ações que poderão ser eficazes para manter o país na liderança mundial de exportação de carne bovina.

e Schuh, G.E. Desenvolvimento da agricultura: estudos de casos. Pioneira. São Paulo. Cap. 5. pp. 257-277.

Miranda, S.H.G. 2001. Quantificação dos efeitos das barreiras não-tarifárias sobre as exportações brasileiras de carne bovina. Tese (Doutorado). Escola Superior de agricultura Luiz de Queiroz. Universidade de São Paulo. Piracicaba. Brasil. 237 pp.

Polaquini, L.E.M., Souza, J.G. de e Gebara, J.J. 2006. Transformações técnico-produtiva e comerciais na pecuária de corte brasileira a partir da década de 90. Rev. Bras. Zootecn., 35: 321-327.

Reis, S.M. dos. 1997. Impacto da política cambial sobre as exportações e os preços de cacau do Brasil, 1997. Dissertação (Mestrado). Universidade Federal do Ceará. Fortaleza. 88 pp.

Silva, C.R.L. da e Carvalho, M.A. de. 1995. Taxa de câmbio e preços de commodities agrícolas. Informações Econômicas, 25: 23-35.

Yokoyama, L.P., Igreja, A.C.M. e Neves, E.M.N 1989. Modelo shift-share: uma readaptação metodológica e uma aplicação para o Estado de Goiás. Em: Congresso Brasileiro de Economia e Sociologia Rural, 27. 1989. Piracicaba. Anais... Sober. Brasília 1: 62-78.

Archivos de zootecnia vol. 59, núm. 225, p. 79. 